\title{
CONE-BEAM ASSESSMENT OF GUTTA PERCHA REMOVAL BY RECIPROC VERSUS PROTAPER UNIVERSAL RE-TREATMENT
}

\author{
Mahmoud M. Badr ${ }^{*}$ and Mohammed Elsayed Rokaya**
}

\begin{abstract}
This study intended: to assess use cone-beam analysis of gutta percha removal by Reciproc versus ProTaper Universal retreatment systems.

Methods: Thirty extracted humanoid lower first molars were collected. The mesiobuccal canals had been mechanically prepared in a crown-down approach using the "TF Adaptive" setting on Elements Motor. TF Adaptive Green ML1 file (Medium Large) and Yellow ML2 size 35 taper 4\% till the file gets to the effective length. the obturation was done by lateral condensation technique with gutta pearch size 35 taper $6 \%$, and $\mathrm{AH}$ plus in the same visit, and using gutta pearch size 25 taper $2 \%$ as auxiliary cones with spreader size 25 . samples were unintentionally split into two investigational groups concurring to the re-treatment method used $(\mathrm{n}=15)$. Group I: Reciproc system, and Group II: ProTaper Universal re-treatment. Cone beam (CBCT) was used to evaluate the gutta percha removal in both groups.
\end{abstract}

Results: little left guttapercha after the wastage of Reciproc kit $\left(\mathbf{0 . 1 6 9 \mathbf { m m } ^ { 3 } )}\right.$ afterwards the Pro Taper kit $\left(1.459 \mathbf{m m}^{3}\right)$. There were little left gutta percha after the use of the Reciproc system (The rate of decrease was $96.82 \%$ ) followed by the ProTaper Universal re-treatment kit (The rate of decrease was $76.17 \%$ ).

Conclusions: Reciproc was more effective than Protaper Universal kit in the elimination of resin sealers like epoxy based sealers and the guttapercha through re-treatment.

\section{INTRODUCTION}

Endodontic catastrophes may arise even after the main typical of procedures was followed ${ }^{(1)}$. Endodontic catastrophes may be recognized to indecorous cleaning and shaping of canal ramifications, practical faults or inability to create hermetic clo- sure, in that way helping persistence or re-growth of microbes in the root canals ${ }^{(2)}$.

Whenever entree to the core canal is possible, endodontic re-treatment should be the preferred choice $^{(3,4)}$. Several techniques for filling materials removal are currently used, including manual and

* Lecturer of Endodontics, Faculty of Oral and Dental Medicine, Future University in Egypt

** Associate Professor, Associate professor of Endodontics, Faculty of Dental Medicine, Al-Azhar University, Assuit, Egypt. 
rotary. However, no re-treatment procedure can eliminate the filling material completely; resulting in residual debris attached to the core canal walls after re-instrumentation ${ }^{(5.6)}$.

Elimination of gutta-percha (GP) is time overwhelming from well packed root canals. Various tools and techniques have been planned to enable elimination of root fillings (gutta-percha and or sealer). These contains endodontic hand instruments with or without gutta percha solvents, rotary instruments, heat carrying instruments and ultrasonic devices. Physical instrumentation for gutta-percha is dreary course ${ }^{(7)}$.

Traditionally solvents were used to get the guttapercha soluble. These includes xylol, tetrahydrofuran and many more ${ }^{(8)}$. NiTi files precisely intended for re-treatment have been presented. Rotating instruments are fewer time intervening than physical for root fillings elimination. Yet, considerable quantity of obturating materials has been observed inside the canals ${ }^{(9)}$.

The proper removal of obturating materials from inadequately prepared and filled root canals requires a substantial effort and can be challenging and timeconsuming. Many studies evaluated the use of reciprocating files to eradicate gutta-percha from the root canals ${ }^{(10,11)}$.

So, the existing study was aimed to usage of cone-beam Computed Tomography analysis of gutta percha elimination by Reciproc versus ProTaper Universal re-treatment kit.

\section{MATERIALS AND METHODS}

30 not long extracted developed humanoid lower six teeth had been gathered from the dental hospital patients, Al azhar, and Future Universities. Within each molar, the MB canals were chosen. molars were chosen in a way that angle of curvature of the Mesial root curvature was ranging 15 and 30 degrees as stated by Schneider technique ${ }^{(12)}$.

\section{Sample preparation}

Teeth were decoronated to equalize and assimilate the WL to $16 \mathrm{~mm}$. K-Flex file \#15 was used to pass beyond the apex to keep the canal patent through out the mechanical procedure

Working length of the mesio-buccal canals was measured by placing a size $10 \mathrm{~K}$ file into the canal till it exited from the apex and apical patency was confirmed; this length was measured, and the working length (WL) was set $1 \mathrm{~mm}$ short of that length to achieve a length.

\section{TF Adaptive canal shaping technique}

Use the "TF Adaptive" setting on Elements Motor.

1. The pulp chamber was flooded with $5.25 \%$ Naocl*.

2. Slowly advance the TF Adaptive Green ML1 file (Medium Large) size 25 taper 8\%. The speed of rotation was $500 \mathrm{rpm}$ and torque were $4 \mathrm{Ncm}$ with a single controlled motion until the file engages dentin then completely withdraw the file from the canal ${ }^{(13)}$.

3. Wipe off the flutes. Deliver $5 \mathrm{ml}$ of $5.25 \% \mathrm{Naocl}$ solution to the pulp chamber.

4. Stages 3 and 4 was repeated using the file ML1 till effective length is attained.

5. Repeat steps 3 and 4 with the Yellow ML2 size 35 taper $4 \%$ until the file reaches working length.

6. The canals were thoroughly irrigated using $2 \mathrm{ml}$ of $2.6 \%$ sodium hypochlorite and $17 \%$ EDTA solution between every successive file. With the aid of a 27-gauge needle** at a depth of $2-3 \mathrm{~mm}$ from the working length. Finally, $5 \mathrm{ml}$ of

\footnotetext{
* Sodium hypochlorite (Clorex) Houshold Cleaning Products, Company of Egypt Ltd, Egypt.

** NaviTip, UITRADENT, USA.
} 
$2.6 \%$ sodium hypochlorite, $5 \mathrm{ml}$ of $17 \%$ EDTA solution, and $10 \mathrm{ml}$ of distilled water were used as a flush of the canals.

7. Paper Points were used to dry the canals.

8. The obturation was done by lateral condensation technique with gutta pearch size 35 taper $6 \%$, and $\mathrm{AH}$ plus endodontic sealer in the same visit, and using gutta pearch size 25 taper $2 \%$ as auxiliary cones with spreader size 25 .

9. The access cavity was temporarily sealed with Cavit temporary filling material, and the stored at $37^{\circ} \mathrm{C}$ in $100 \%$ humidity for two weeks to allow complete setting of sealer.

\section{Root canal re-treatment}

Prepared samples were unintentionally separated into two investigational groups $(n=15)$ rendering to the re-treatment kit utilized. An anonymous investigator lacking the knowledge of the aim of this study, hold the teeth sampling that were placed in an empty enfold by selecting randomly and placing them into one of the experimental groups. Also, an endodontist with high level experience had undergone the root canal retreatments for all the teeth in the experimental groups.

\section{G1: Reciproc system}

Using the 0.06 tapered file with size \#40 called $\mathrm{R} 40$ file with their unique motorized contra angle in the golden motor from VDW utilizing the reciprocating kinematic mode. The file had been progressing towards the apex in a picking motion with a striking wave each with around 2-3 $\mathrm{mm}$ with caution. Rendering to the company order, mild force towards the apex was done utilizing a sweeping motion across the canal dentin walls. Once the striking waves are done, the file was uninvolved out of the root then properly wiped and cleaned from dentin debris that might be present within and between the flutes, then the canal was flooded with $5.25 \% \mathrm{NaOCl}$ was inserted into the canal by plastic syringe and a side vented needle This process had been recurrent until the file accomplished effective appropriate WL.

\section{G2: ProTaper Universal retreatment kit";}

The running specifications had been set as follows: $350 \mathrm{rpm}$ speed and, $2.6 \mathrm{~N}$ torque. The first file called (D1) for elimination of the filling out from the orifice apically till the coronal third of the canal, however the next files in the retreatment kit called (D2\&D3) were used afterwards while progressing apically with the same specifications and motion kinematics with caution till the guttapercha along the whole length of the canal was efficiently eliminated. Afterwards, (X3 \#30 \&X4 \#40) rotary files were appropriately utilized after the retreatment kit till the appropriate WL of the canal. During the root canal instrumentation and between each rotary file and the other, $\mathrm{NaOCl} 5.25 \%$ irrigation was properly used using a plastic syringe and side vented needle for flushing out of debris and to avoid pushing of debris beyond the apex.

Around 15-20 mL of irrigation had been consumed throughout the re-treatment procedure. Every file had been utilized for the re-treatment and mechanical preparation of only 3 canals. Retreatment had been well-thought-out ample when every file had been successfully accomplished the appropriate effective WL for 5 times. Following the instrumentation, irrigation using $5.25 \% \mathrm{NaOCl}$ was done. Afterwards, EDTA as a chelating agent (15\%) was placed for 2 minutes then flushed with the irrigant. Finally, drying the canal with properly fitted paperpoints. ${ }^{(14)}$.

\footnotetext{
* VDW GmbH, Munich, Germany.

** Dentsply Sirona Endodontics.
} 


\section{Cone beam (CBCT) assessment:}

A JMorita RS250 CBCT - was used to scan the samples at 2 phases (afterwards filling of the canal with guttapercha and afterwards elimination of the guttapercha) (Fig 1). The examination has been finished with a field of view (FOV) of 250 mmxHOR $95 \mathrm{~mm}$. The concentration were then reassembled with.354 mm iso-metric voxel size. The volt of the tube was $85 \mathrm{kVp}$ and $9 \mathrm{~mA}$ and the contact was $10 \mathrm{sec}$.

\section{D analysis of the volumetric images:}

Following gaining of cone beam CT pictures, those di-com files were introduced within a copy examination osirix program ${ }^{* *}$. However as a trial to precisely differentiate between the guttapercha core material and the canal walls, the sharpness-contrast range was modulated within the average threshold as recommended by the system. (Fig 1). This ranging level was provided for all specimens in sake of systematizing and standardizing the measuring technique. Utilizing this program, area of curiosity was shaded by marking the outer borders of the core at 4 to 6 parts through out the whole length.

This program utilize those amounts to sum and figure the remaining core volume. That had been calculated mm3 (Fig 1). By the same way using the system, the remnants of guttapercha filling material were computed. The percentage of volume of residual core filling was calculated with this equation:

\section{Statistical analysis:}

The decline of the volume of the core filling following every re-treatment procudure was evaluated using the Kraskul--Willis testing \& addition box \& wheskar ploting. the P-value of $<0.05$ was measured showing statistic important. The arithmetical investigates were achieved using IBM VLDR software $41.0^{* * *}$.

* JMORITA MFG. CORP. Kyoto Japan.

** Osirix 32-bit, Geneva, Switzerland.

*** IBM, Armonk, New York, NY, USA.

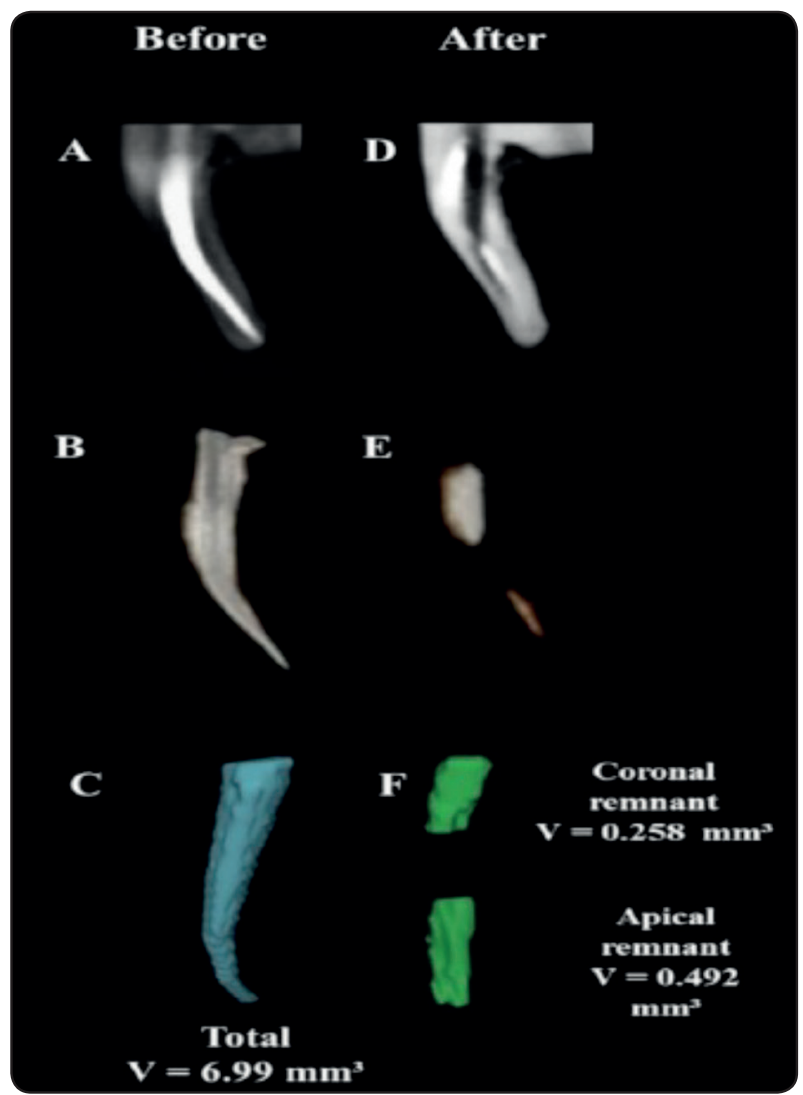

Fig. (1): ConebeamCT displaying the measuring technique of the size of filling material.

\section{RESULTS}

The existing study exhibited that those two techniques are efficient in eliminating the obturating core substances, however both of them was able to totally eliminate the filling substances. The sum of the root core filling in the root canals sign. declined after the two re-treatment protocols $(\mathrm{P}<0.05)$. Moreover, there was no statistically important differences $(\mathrm{P}>0.05)$ between the obturating substances volumes lasting amongst these two groups after re-treatment as seen in the table \#1

It was demonstrated that there was little remnants of obturating substances after utilizing the Reciproc rotary files (0.169) then by the ProTaper re-treatment kit (1.459) (Fig 2). 
TABLE (1): The primary bulk of the obturating material (in $\mathrm{mm} 3$ ) and the remnants volume (in mm3) following the re-treatment techniques, and the ratio of decline (\%) in the obturating substances.

\begin{tabular}{|c|c|c|c|c|}
\hline & $\begin{array}{c}\text { The mean of initial bulk of the filling } \\
\text { material (in mm) }\end{array}$ & $\begin{array}{c}\text { the remaining } \\
\text { volume }\end{array}$ & $\begin{array}{c}\text { The rate of decrease } \\
(\%)\end{array}$ & P value \\
\hline Reciproc & $5.561 \pm 0.17$ & $0.169 \pm 0.21$ & 96.82 & $P<0.05$ \\
\hline $\begin{array}{c}\text { ProTaper Universal } \\
\text { retreatment }\end{array}$ & $7.220 \pm 0.14$ & $1.459 \pm 0.25$ & 76.17 & \\
\hline P value & \multicolumn{2}{|c|}{$P>0.05$} & \\
\hline
\end{tabular}

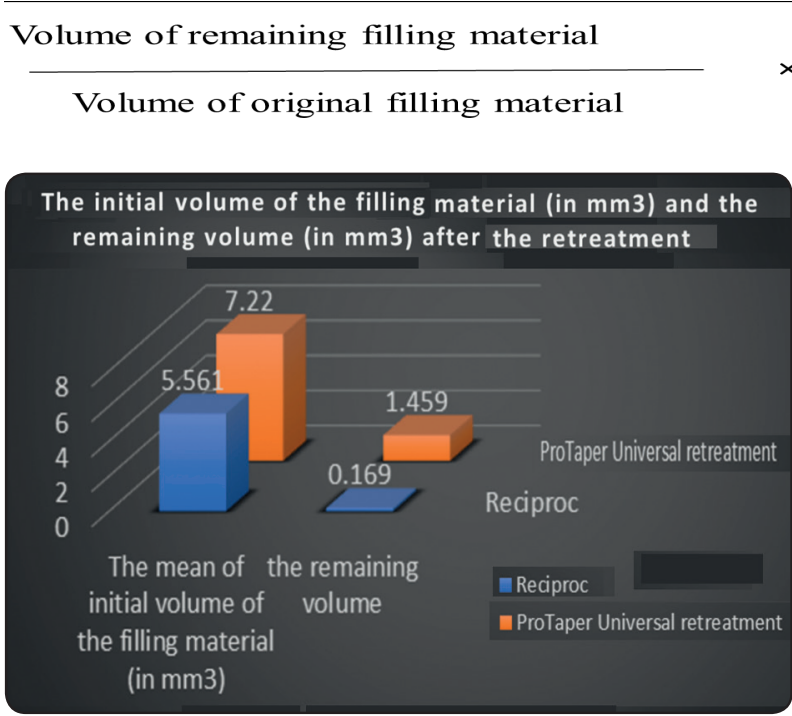

Fig.(2) Showing the primary volume of the obturating substances (in mm3) \& the remnant volume (in $\mathrm{mm} 3$ ) following the re-treatment regimns, and the ratio of decline $(\%)$ in the obturating material.

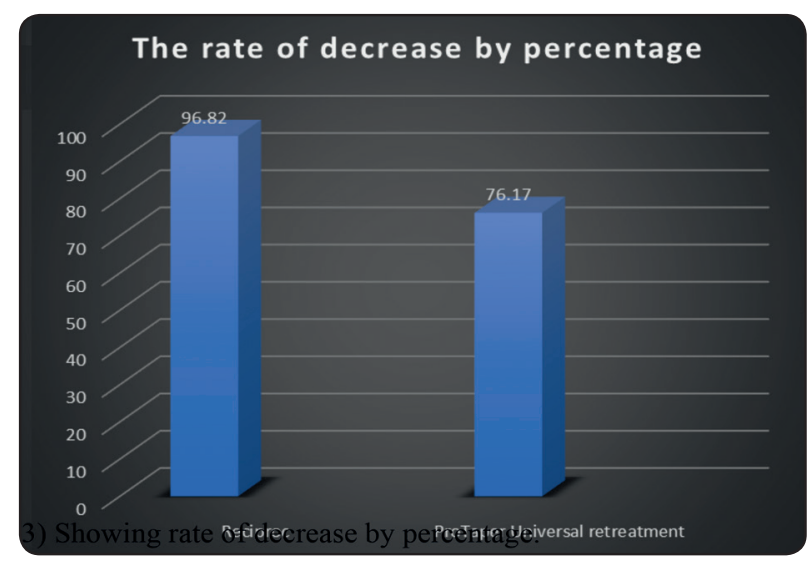

Fig.(3) Showing rate of decrease by percentage. $\times 100 \%=$ volume $\%$ of remaining filling material

Also, it was declared that there was little remnants of obturating substances after utilizing Reciproc rotary kit (The rate of decrease was 96.82\%) then by the ProTaper re-treatment kit (The rate of decrease was $76.17 \%$ ) and (Fig3).

On other hand, it was declared that there was little remnants of obturating substances following utilizing Reciproc kit $\left(0.169 \mathrm{~mm}^{3}\right)$ then by the ProTaper re-treatment kit $\left(1.459 \mathrm{~mm}^{3}\right)$ (Fig 3).

\section{DISCUSSION}

The elimination of obturating substances while the re-treatment allows the files and irrigation solution to deeply involve most of the inaccessible regions of the system, aiming for improved cleansing ability and disinfecting the root canal system efficiently ${ }^{(15)}$. No current re-treatment practice can eliminate root core filling, with the proportion of left behind material oscillating from $45.4 \%$ to $0.02 \%{ }^{(16)}$.

Moreover in our study, the total elimination of the obturating materials and components; resin based sealer and gutta-percha filling material utilizing different kinematics through both full rotation or reciprocation with final flush irrigation regimen was not properly accomplished.

Utilizing the reciprocation kinematics with Reciproc rotary files ended up with the smallest 
quantity residue obturating core material after retreatment $\left(.169 \mathrm{~mm}^{3}\right)$. Also, the motion kinematics utilizing the reciprocation motion in the Reciproc rotary files kit resulted in the best results in terms of re-treatment., with a mean obturating substances decline ratio (97.62 \%). Multiple studies have detected that utilizing the reciprocation kinematics in root canals for re-treatment procedures resulted in better and more successful outcome than the full rotation kinematics ${ }^{(10,14)}$. Also, the capabilities of such rotary files utilizing reciprocation motion kinematics like Reciproc kit is may be due to its unique $\mathrm{S}$-shaped cross sectional design accompanied with double edged sharply cutting edges with increased +ve angle of cutting. Also its unique design allows more space for debris removal due to increased clearance space, so less dentin debris lodging in the apical root canal area and little debris passing beyond the apex with superior results and better prognosis. ${ }^{(17)}$.

On other hand the cross sectional plan had an excessive effect on the sharpness effectiviness accompanying the rotary files. That's why, its concluded that the unique design of the Relciproc files plays an important and primary role in the retreatment efficiency when in comparison with the triangular cross sectional diameter of the PT rotary kit. ${ }^{(18)}$. Disagreement with the outcoming results of our study, there were no major differences between both files with different motion kinematics in different studies in different canal cross sections. ${ }^{(19)}$.

In addition, (15) another study showed that there were no big differences in the elimination capabilities of the obturating materials of the root canals between both kinematics reciprocation and full rotation motions.. This different outcomes between the motion kinematics may be due to different canal cross sections like oval or curved or even round canals, also either straight or curved canals. Those variables may be the reason behind the conflict in the study outcomes. Also, the preoperative status of the obturated teeth in terms of quality and type of the obturating substances and either utilizing a gutta-percha solvent materials or not. In our study, only lower $1^{\text {st }}$ premolars with straight oval root canals that were obturated use up the cold lat. compression method were utilized.

The outcomes of our study are in harmony with these offered from other authers like Bernardes et al. ${ }^{(14)}$, utilized a parallel technique procedure \& stated quantity of residue obturating materials and substances ( Gutta-percha with $\mathrm{AH}+$ utilizing the cold lat comp with the thermo-mechanical obturation compaction techniques) was greatly reduced by the use of the recirproaction kinematics in re-treatment utilizing the Reciproc-R40 instrument in comparison to Protaper Universal re-treatment kit (D1 to F40).

\section{CONCLUSION}

Reciprocation kinematics like Reciproc files were more efficient than the protaper universal system in re-treatment and elimination of obturating materials in terms of gutta-percha and resin based sealers duting the re-treatment procedures.

\section{REFERENCES}

1. Fenoul G, Meless GD, Perez F. The efficacy of R-Endo rotary NiTi and stainless-steel hand instruments to remove gutta-percha and Resilon. Int Endod J. 2010; 43:135-41

2. Marfisi K, Mercade M, Plotino G, Duran-Sindreu F, Bueno R, Roig M. Efficacy of three different rotary files to remove gutta-percha and Resilon from root canals. Int Endod J. 2010; 43:1022-1028.

3. Del Fabbro M, Taschieri S. A systematic review on the outcome of surgical vs non-surgical procedure for the retreatment of periapical lesions. Minerva Stomatol 2007; 56:621-32.

4. Kvist T. Endodontic retreatment. Aspects of decision making and clinical outcome. Swed Dent J Suppl 2001; 144:1-57.

5. Bramante CM, Fidelis NS, Assumpção TS, Bernardineli $\mathrm{N}$, Garcia RB, Bramante AS, et al. Heat release, time required, and cleaning ability of MTwo $\mathrm{R}$ and ProTaper universal retreatment systems in the removal of filling material. J Endod 2010; 36:1870-3. 
6. Jantarat J, Malhotra W, Sutimuntanakul S. Efficacy of grapefruit, tangerine, lime, and lemon oils as solvents for softening gutta-percha in root canal retreatment procedures. J Investig Clin Dent 2013; 4:60-3.

7. Horvath SD, Altenburger MJ, Naumann M, Wolkewitz M, Schirrmeister JF. Cleanliness of dentinal tubules following gutta-percha removal with and without solvents: a scanning electron microscopic study. Int Endod J. 2009; 42:1032-8.

8. Hülsmann M, Stotz S. Efficacy, cleaning ability and safety of different devices for gutta-percha removal in root canal retreatment. Int Endod J. 1997; 30:227-233.

9. Gu LS, Ling JQ, Wei X, Huang XY. Efficacy of ProTaper Universal rotary retreatment system for gutta-percha removal from root canals. Int Endod J. 2008; 41:288-95.

10. Zuolo AS, Mello JE Jr, Cunha RS, et al. Efficacy of reciprocating and rotary techniques for removing filling material during root canal retreatment. Int Endod $\mathbf{J}$ 2013; 46:947-53.

11. Silva EJ, Orlowsky NB, Herrera DR, et al. Effectiveness of rotatory and reciprocating movements in root canal filling material removal. Braz Oral Res 2015; 29:1-6.

12. Schneider SW. Comparison of the canal preparation in straight and curved root canals. Oral Surg 1971; $32: 271-5$.

13. Nair PN, Henry S, Cano V, Vera J, Microbial status of apical root canal system of human mandibular first molars with primary apical periodontitis after "one-visit" endodontic treatment. Oral Surgery, Oral Medicine, Oral
Pathology, Oral Radiology, and Endodontology, 2005. 99:231-252.

14. Bernardes RA, Duarte MA, Vivian RR et al. Comparison of three retreatment techniques with ultrasonic activation in flattened canals using micro-computed tomography and scanning electron microscopy. International Endodontic Journal 2016; 49:890-7.

15. Rossi-Fedele G, Aly Ahmed HM. Assessment of root canal filling removal effectiveness using micro-computed tomography: a systematic review. Journal of Endodontics 2017;43: 20-6.

16. Yilmaz F, Koç C, Kamburoğlu K et al. (2018) Evaluation of 3 different retreatment techniques in maxillary molar teeth by using micro-computed tomography. Journal of Endodontics 2018;44: 480-4.

17. Plotino G, Giansiracusa Rubini A, Grande NM, Testarelli L, Gambarini G (2014a) Cutting efficiency of Reciproc and WaveOne reciprocating instruments. Journal of Endodontics 2014;40: 1228-30.

18. Bürklein S, Hinschitza K, Dammaschke T, Schäfer E (2012) Shaping ability and cleaning effectiveness of two single-file systems in severely curved root canals of extracted teeth: Reciproc and WaveOne versus Mtwo and ProTaper. International Endodontic Journal 2012;45: 449-61.

19. Monquilhott Crozeta B, de Sousa-Neto MD, Leoni GB et al. A micro-computed tomography assessment of the efficacy of rotary and reciprocating techniques for filling material removal in root canal retreatment. Clinical Oral Investigation 2016; 20: 2235-40. 